\title{
Research on Knowledge Innovation in Quality Management Process
}

\author{
Chai Congliang \\ Aviation University of Air Force \\ Jilin Changchun China \\ email:myg123789@sina.com
}

\author{
Mu Yonggui \\ Aviation Theory Department of Aviation University of \\ Air Force \\ Changchun China \\ email:myg123789@sina.com
}

\begin{abstract}
At present, application of knowledge in the field of quality management theory and method can achieve improved quality and innovation. The primary means of knowledge innovation in quality management process are knowledge value chain and the quality knowledge innovation of the organizational knowledge fermentation model. Knowledge innovation provides highly complicated work with an effective quality assurance.
\end{abstract}

Keywords-quality management; knowledge management; knowledge innovation; knowledge value chain; knowledge fermentation model

I. THE APPLICATION OF KNOWLEDGE MANAGEMENT HAS ACHIEVED IMPROVEMENT OF QUALITY AND INNOVATION IN THE PROCESS OF QUALITY MANAGEMENT

\section{A. The operation on quality management business is a} process of knowledge accumulation and learning

Fine in the early 20th century has carefully studied the relationship between quality and learning and created an analysis model researching on the failure and accordance cost. He found that the best level of quality increased with learning time. ${ }^{[1]}$ Dada and Tapiero analyzed and researched respectively the learning effect in the quality control ${ }^{[2-3]}$, reaching the same conclusion. Sitkin and Clarke had an empirical study, research and analysis on the knowledge or learning activities in the quality management and concluded that the traditional TQM had too much emphasis on quality control and was not suitable for highly complicated work. Thus, they had knowledge management applied into the quality management process and re-built the TQM theoretical framework. ${ }^{[4]}$ Clarke pointed out that the knowledge management and two quality system frameworks---Baldridge National Quality Award and ISO9000 standard system---are closely related, and compared the different characteristics of the two systems and the knowledge management. Many scholars have demonstrated the characteristics of quality knowledge management and the relations with quality management, and from the perspective of knowledge management, outlined and analyzed the quality management work. Practice has proved that the quality of the management work in organizations contains a large amount of intellectual activities and is a process of knowledge management and organizational learning. Quality experts analyzed on the knowledge fermentation process, fermentation type and function in the organization quality management work, and were more convinced than before that the operation on quality management itself was a process of knowledge accumulation and learning.

B. The transformation of implicit and explicit knowledge into each other promotes the improvement of the quality management performance

Doolev pointed out, "because the most fundamental value of an enterprise depends on the knowledge that can not be imitated, the implicit knowledge that is difficult to imitate will become more and more important. Therefore, we can expect the quality management system to focus its attention on implicit knowledge." ${ }^{[5]} \mathrm{K}$. Linderman et. al by using the related theories of Nonaka's process of knowledge innovation, point out that quality management is the source to generate knowledge innovation and knowledge innovation, in turn, promote quality management and finally improve organizational performance. The relevant mechanism is also elaborated in detail.

1.3 Knowledge management methods are the accelerator for quality management

Jae Kyeong Kim et. al, in the process of product design, adopt knowledge management to classify and manage the HOQ chart in QFD, which has greatly improved the efficiency and effectiveness of the HOQ chart. ${ }^{[6]}$ Demet Bayraktar, by establishing a knowledge-based expert systems to examine some elements in quality assurance system, ${ }^{[7]}$ such as the "contract review" process in ISO9000, and "procurement" procedures, etc. It highly efficiently ensures the the normal operation of the ISO9000 quality to assurance system. Larry Todd Wilson and Diane Asay, through the study of quality management trends, pointed out that quality management needed to adapt to the needs of the knowledge economy. Thus the CMM was born, and they compared quality management practices with the CMM, demonstrating that CMM was a standard to effectively solve quality problems in enterprises in the era of knowledge economy. ${ }^{[8]}$ In fact, it is just the use of knowledge management methods and tools that it ensures high-quality knowledge assets innovation. Some scholars have systematically analyzed the characteristics of the series of standards of the ISO9000 quality management and quality assurance from the angle of knowledge structure and hierarchical structure, which offers a new way of thinking for people to understand the standard. This undoubtedly is a new attempt to use knowledge management methods to the complicated system of ISO9000. Paige Leavitt discusses the relationship between $6 \sigma$ and knowledge management, by comparing the process of the DMAIC of $6 \sigma$ and the knowledge management process of 
APQC, they point out that the use of $6 \sigma$ DMAIC method can help enterprises to carry out knowledge management more quickly and better, and at the same time they analyzes the knowledge innovation process in the implementation of $6 \sigma$, making enterprises recognize that there are similarities and complementary function in the way of the methods of the two. ${ }^{[9]}$

\section{MAIN METHODS IN KNOWLEDGE INNOVATION IN THE} PROCESS OF QUALITY MANAGEMENT

\section{A. Quality knowledge value chai}

"Knowledge value chain" was originated from "value chain" in competitive advantage written by Michael Porter (Michael E. Porter) in 1985. in the proposed The traditional value chain model was built on the basis of material factors, discussing the process of profit growth. In the knowledge economy society, knowledge capital becomes the most important resource, and the traditional value chain model due to its neglecting the discussion of the added value of knowledge, has been unable to meet the needs of enterprises. Therefore, to explore how knowledge grows in a variety of functions in the enterprise activities becomes an urgent subject. Weggeman thinks that the knowledge value chain is composed of four consecutive process as knowledge acquisition, knowledge integration, knowledge innovation and knowledge diffusion. ${ }^{[10]}$ Domestic scholars recognized the knowledge value chain similar with it in general, including knowledge acquisition and processing, knowledge sorting and storage, knowledge dissemination and sharing and knowledge innovation and application. These views have neglected an important part of knowledge protection and environmental factors that promote their progress. We believe that the main aspects of the enterprise quality knowledge value chain should include five aspects as quality knowledge acquisition, quality knowledge sharing, quality knowledge innovation, quality, knowledge protection and quality knowledge application. Besides, quality knowledge value chain should also include auxiliary sections of quality culture and information technology. To synthesize these aspects of function can improve knowledge sharing and the efficiency and effectiveness of knowledge innovation. Specific description of quality knowledge value chain is shown as Figure 1. Quality knowledge acquisition is the foundation, quality knowledge sharing is a necessary means, quality knowledge innovation is the source of new knowledge, quality, knowledge protection is the guarantee, quality knowledge application is the purpose and way to realize value of quality knowledge management, and quality culture and information technology knowledge is the necessary environment to realize flow of the quality knowledge value chain.

In fact, the quality knowledge innovation is inherent in the whole process of quality knowledge aquisiton, sharing and application. It achieves the goals of creating added value of knowledge by the pursuit of new discoveries, exploring new law and the accumulation of new knowledge. Quality knowledge innovation includes not only the quality of products and innovation of level of knowledge services, but also quality management quality and innovation of knowledge improvement capacity. We can guide enterprise quality innovation by using SECI knowledge innovation model pointed by Nonaka in 1994 .

- Socialization. discussion of quality problems, exchange of quality experience, quality behavior imitation and customer contact.

- Externalization. quality knowledge coding, quality knowledge documentation and establishment of quality management system.

- Combination. quality management training, quality statistical analysis, upgrading of quality management system, and improvement of quality tools.

- Internalization. application experience of quality of knowledge, quality program practice, and quality issues solving.

\section{B. Quality Knowledge Innovation of Organizational Knowledge Fermentation Model}

Based on the assumptions of knowledge nature, genetic variability, group nature, the media, He Jinsheng proposed the knowledge fermentation model in organizational learning. ${ }^{[11]}$ In the knowledge fermentation model, knowledge fermentation mainly concern some factors influencing on the growth of knowledge as "knowledge strain", "knowledge matrix", "knowledge enzyme in knowledge matrix" and "knowledge fermenting bar". To take QC team as an example, knowledge innovation in the QC group organizational learning process is expounded.

- Quality Knowledge Strain. How to make the right quality improvement and innovation needs by enterprises is the starting point of the generation of quality improvement and innovation. The process to find the demand can be called the discovery process of knowledge strain. These quality knowledge strains are not generated out of thin air, but closely related with the organizational activities such as periodic quality group activities, quality meeting, solving product quality problems from customers' complaints and the pursuit of $6 \sigma$ achieving zero defect and so on.

- Identification and Improvement of Quality Knowledge Matrix. With the task of quality improvement or demand, how does the QC team achieve quality innovation goals? Obviously, randomly picked up QC team may not have the capacity for innovation. The composition of QC team is the key to quality innovation. The concept of knowledge body lies in proposing a clear policy, requiring the knowledge composition and quality of learning of members to be able to overcome difficulties. The QC team can improve the quality of matrix through training, expert guidance, open composition mechanism.

- (3) Quality Knowledge Enzymes. Having the expert staff coming from different departments, with different professional background, it is another key to complete quality innovation about how to 
coordinate the work in the QC team. The QC team as a learning type necessarily requires a flat organizational structure. And this can be filled with the spirit of democracy, fully mobilize the enthusiasm of each person. We can consider the development of people who are prestigious, competent, not subjective, with background knowledge of management and psychology as the backbone of organizational learning. He will play a strong inspiration, induction, encouragement role to achieve the quality innovation. The factor can have the role in organizational learning as organization, mediation, coordination, communication and encouragement is called enzyme. In order to promote quality knowledge fermenting, the organization should take particularly attention to the role of coordination and catalysis of quality managers.

- Quality Knowledge Fermenting Bar. Quality knowledge fermenting bar refers to the gathering of within and outside of the organization to have quality knowledge "group gathering" place and mechanism. Knowledge fermenting members under different situations may have different objectives and gathering, forming different fermentation types as digested type of fermentation, adaptive type of fermentation campaign type of fermentation, evolution type of fermentation and re-shape type of fermentation. In the process of the QC team spreading experience and knowledge, knowledge receivers required to combine foreign experience with their own knowledge and experience. They chew on and understand the knowledge, making it "mature" knowledge so as to achieve proper use of knowledge. That is digested type of fermentation. Adaptive type of fermentation means that QC team can not simply apply to the quality management experience in other enterprises; they must set up an implementation standard and combine the quality management system principles with enterprises practices so as to achieve value-added effectiveness to enterprises in ISO9000. Campaign type fermentation means that QC group gathering internal and external multi-disciplinary expertise, including suppliers and customers, to jointly explore enterprise and technological issues and solve enterprise problems, generating new programs, new methods and new knowledge. Evolution of type fermentation means that as strains survive of the fittest in the environment changing, knowledge is evolving. One can not have the experience, technology and methods rest on its laurels. If the implementation of ISO9000 is an adaptive type of fermentation, the upgrading of ISO9000 standard, as from version 1994 to 2000, is an evolution type of fermentation. The re-shape type of fermentation means that fermentation process can produce new species and substances. Some fundamental innovation of QC group is the result of knowledge fermentation. For example, the pointing out of ISO9000 belongs to innovative fermentation. Although the establishment of quality system practices and theories has made significant progress, as a new management techniques and the establishment of certification system achieves a qualitative leap.

\section{CONCLUSION}

Knowledge management applying to quality management is the inevitable result of quality management adhering to continuous improvement and the pursuit of excellence. Knowledge management achieves improvement of quality and innovation. Practice has proved that the quality management is in fact a process of knowledge management and innovation. The further development of quality management needs continuous quality improvement. The primary means of knowledge innovation of quality management is quality knowledge value chain and organizational knowledge fermentation model. Using quality knowledge value chain to analyze the process of knowledge acquisition, sharing, innovation and protection in quality management, can effectively promote quality improvement and innovation. Using organizational knowledge fermentation model to explain mechanism of knowledge innovation, development and diffusion in quality management can provide a clear idea for quality management and improvement innovation meanwhile, provide a reference for the quality core strategies of enterprises.

\section{REFERENCES}

[1] Fine, C.H. Quality improvement and leaming in productive systems[J]. Management Science, 1986. 32(10): 1301-1315.

[2] Dada, M. Marcellus, R.Process control with leaming[J]. Operations Research. 1994, 42(2): 323-336.

[3] Tapiero, C.S. Production learning and quality controI[J]. IIE Transactions. 1987. 19(4): 362-370.

[4] Sitkin, S.B.,Sutcliffe,K.M.,Schroeder R.G..Distinguishing control from leaming in total quality management:A contingency perspective[J]. Academy of Management Review, 1994.19(3): 537-564.

[5] Doolev, K.J. The paradigms of quality: Evolution and revolution in the history of the discipline: Advances in the management of organizational quality[M]. Greenwich, CT: JAI Press. 2000.

[6] Kim, J.K., Han. C.H.,Choi, S.H.,et al.A knowledgebased approach to the quality function deployment[J]. Computers \& Industrial Engineering. 1998, 35(1): 233236. 